Originalveröffentlichung in: Zivie-Coche, Christiane und Guermeur, Ivan (Hg.), "Parcourir l'éternité", Hommages à Jean Yoyotte Bd. 2 (Bibliothèque de l'École des Hautes Études, Sciences Religieuses 156), Turnhout 2012, S. 757-775

\title{
DER GROSSE REPITHYMNUS IM TEMPEL VON ATHRIBIS
}

\author{
Christian LEITZ*
}

Im östlichen Umgang L 1 des großen Tempels von Athribis befindet sich im 1. Register der inneren (westlichen) Seite in zehn Feldern ein Hymnus an die Göttin Repit ${ }^{1}$. In den ersten vier Feldern werden ihre Erscheinungsformen in Oberägypten genannt, in den nächsten beiden die in Unterägypten, die letzten vier besitzen keine geographische Ordnung mehr. Jedes Feld besteht aus vermutlich 13 Zeilen, von denen die obersten in keinem Fall erhalten sind, und einer langen senkrechten Zeile, wo vielleicht [wdn $n]$ Rpwt irt $\mathrm{Hr}$ hr imntt: "[Umlaufopfer für] Repit, das Horusauge im Westen“" stand. Geographisch geordnete Listen von Göttinnen sind deutlich seltener als solche von Göttern, außerhalb von Dendara sind bislang keine bekannt geworden ${ }^{2}$. Es handelt sich somit um einen Text, der den zu Ehrenden sicherlich interessiert hätte und der hier vorab schon einmal mit einem kurzen inhaltlichen Kommentar bekannt gemacht werden soll, die endgültige Publikation wird dann im Rahmen der Athribis-Reihe erfolgen.

\footnotetext{
* Professor für Ägyptologie an der Universität Tübingen, derzeit Direktor der Abteilung für Ägyptologie, Institut für die Kulturen des Alten Orients (IANES).

Der Autor bedankt sich bei Daniela Mendel für das mehrfache gemeinsame Kollationieren des Textes, bei Alexa Rickert für einige Hinweise vor Ort und den Teilnehmern der 3. Ptolemäischen Sommerschule 2009 in Freudenstadt, die an entsprechender Stelle namentlich genannt sind.

1. Vgl. zur Lage den Tempelplan in Chr. Leitz - D. Mendel - Y. El-MasRY, Athribis II, Le Caire, 2010, S. XLVIII (der Hymnus beginnt gegenüber der Säule Y 9 und endet gegenüber Säule Y 6). Zu den geographischen Texten siehe zuletzt J. Fr. QuAcK, „Geographie als Struktur in Literatur und Religion“, in F. Adrom u.a. (eds), Altägyptische Weltsichten ( $\ddot{A} A T$ 68), Wiesbaden, 2008, S. 131-157.

2. Im einzelnen handelt es sich um S. CAuville, Dendara, Le temple d'Isis, Le Caire, 2007, 11, 6 - 12, 12 und 13, 3-10 sowie 14, 3-13; S. Cauville, Le temple de Dendara. La porte d'Isis, Le Caire, 1999, 19, 5 - 24, 15; Dendara I, 91, 11 - 96, 12 und 123, 8 - 128, 9; Dendara XII, 59, $15-81,8$ und 188, 12 - 201, 12; Mam. Dendara, 101, 18 - 103, 13 und 122, 15 - 124, 15.
} 


\section{Feld des Hymnus}

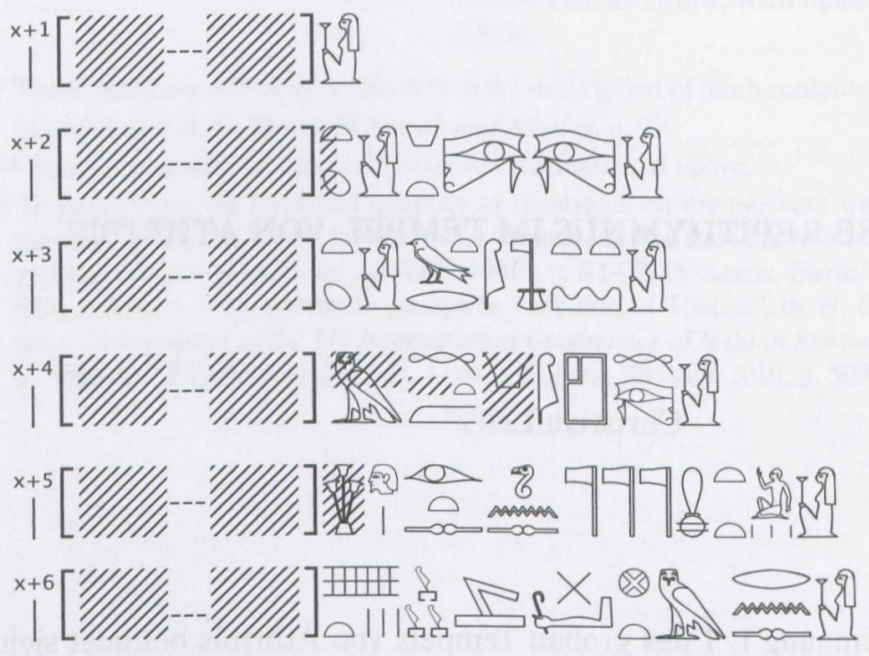

$\mathrm{x}+1 \quad[\ldots]$

$\mathrm{x}+2 \quad[. .$.$] hnw w$ t $w$ dity

$\mathrm{x}+3 \quad[.$.$] wrt m$ hrt-ntr

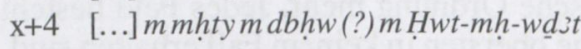

$\mathrm{x}+5 \quad$ [...] hij irt.s nr n.s ntrw mitt rmt

[...]

[...] Herrin der Udjataugen ${ }^{\mathrm{a}}$.

[...] die große [...] in der Nekropole ${ }^{b}$.

[...] im Norden mit den Bestandteilen im Haus des Füllens des Udjatauges ${ }^{c}$.

[...] hinter dem, was sie tut (?) ${ }^{\mathrm{d}}$, vor der sich gleichfalls Götter wie Menschen fürchten ${ }^{\mathrm{e}}$.

x+6 [...] spswt gsw-prw grg niw(w)t $m$ rn.s [...] die die Gaue und Tempel [...], in deren Namen die Städtef ${ }^{f}$ gegründet sind.

a. Nicht in Chr. LeITz (Hg.), Lexikon der ägyptischen Götter und Götterbezeichnungen (OLA 110-116, 129), Leuven, 2002-2003 (im folgenden $L G G$ ).

b. Nach $L G G$ II, 499a kommen Hkzt-wrt-m-hrt-ntr (V, 541b; nur ein Beleg: P. DU BOURGUET, Le temple d'Hathor à Deir el-Médineh [MIFAO 121], Le Caire, 2002, 154 als Bezeichnung der $H w t-H r$-Nbt-htpt) und Špst-wrt-m-hrt-ntrr (VII, 57c; nur ein Beleg: TT 21: N. DE GARIS DAVIES, Five Theban Tombs [ASE 21], London, 1913, Tf. 19 als Bezeichnung der Renenutet) in Frage.

c. Nicht bei Gauthier, DG. Das Toponym wird jedoch noch einmal erwähnt in Athribis in M 1, 52 in der Rede des Horus.

d. Oder ḩ ir sy: ,hinter ihrem Schöpfer" (vgl. einige mögliche Kombinationen in LGG I, 489b).

e. In Dendara XI, 200, 2 ist nr n.s ntrrw eine Bezeichnung der Nechbet. Die Konsequenz könnte eine mögliche Lokalisierung dieser Zeile im 3. o.äg. Gau sein.

f. Diese Übersetzung stützt sich vor allem auf den möglichen Parallelismus mit spswt und gsw-prw. Eine ganz andere Möglichkeit wäre, Niwt: „Theben“" zu lesen, was die vorliegende Zeile im 4. o.äg. Gau lokalisieren würde. Letzteres würde von der Position her gut passen. 


\section{Feld des Hymnus}

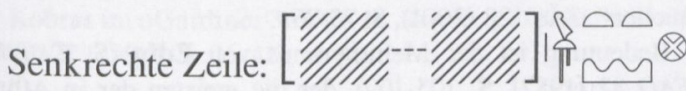
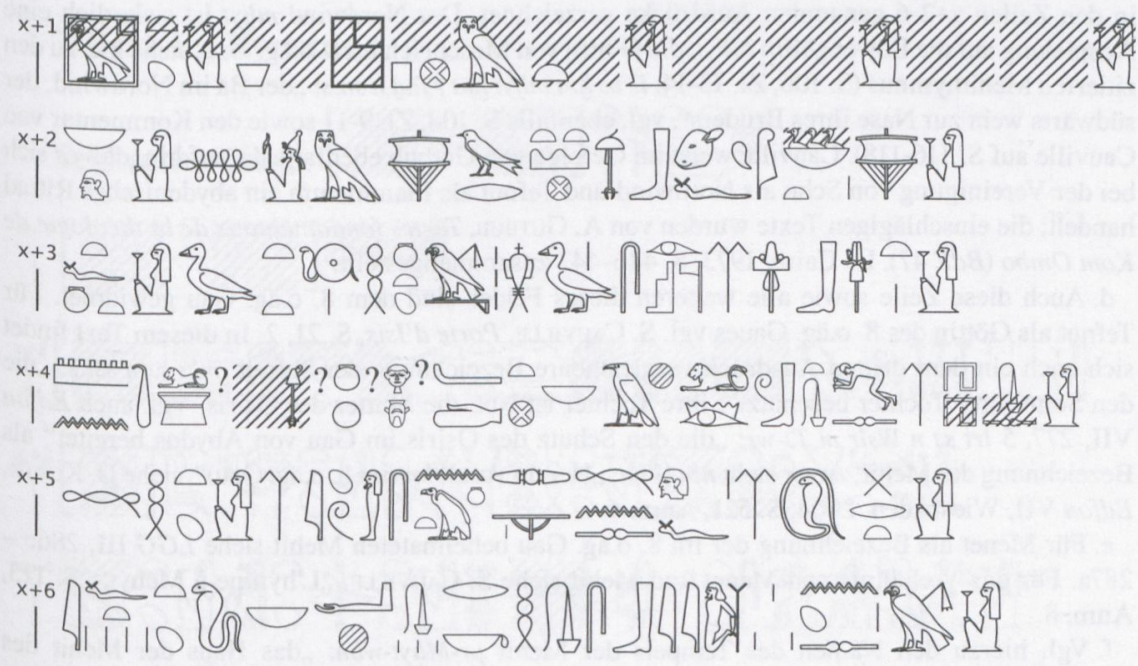

Senkrechte Zeile: [...] imntt: [...] Westen.

$\mathrm{x}+1 \quad H w t-H r[\ldots] H w t-[s h m]$ wrt $[\ldots] \quad$ Hathor $[\ldots] \mathrm{Hu}^{\mathrm{a}}$, die Große [...].

X+2 nbt tp su Wsir $m$ T3-wr wbst fnd.f $m$ Die Herrin des Kopfes ${ }^{b}$, der Schutz des mhyt

Osiris im Gau von Abydos, die seine Nase öffnet als Nordwind ${ }^{c}$.

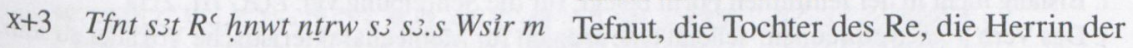
sbdw

Götter, der Schutz ihres Sohnes Osiris in Abydos $^{\mathrm{d}}$.

x+4 Mnt wbnt hryt-ib Bhdt nbt sht msit Menet ${ }^{e}$, die Aufgehendef, die inmitten von hsst wbdt hftyw.s $m$ hh.s

Behedet (im 8. o.äg. Gau) ${ }^{\mathrm{g}}$ ist, die Herrin

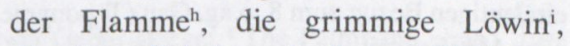
die ihre Feinde mit ihrem Gluthauch verbrennt.

x+5 Mhyt $m$ irw.s $n$ mwt nt Mnw mhn.s Mehit in ihrer Gestalt als Mutter des Mink, tp.fm mhnyt wrt

$\mathrm{x}+6$ lfdwt nbwt 'hw rkhwt sbiw nw s3.s $\mathrm{Hr}$ die ihre Position auf seinem Kopf eingenommen hat als große Umringlerschlange!. Die vier Kobras $^{\mathrm{m}}$, die Herrinnen der Feuerbecken, die die Feinde ihres Sohnes Horus verbrennen.

a. Angesichts der Zugehörigkeit zum 7. o.äg. Gau die wahrscheinlichste Ergänzung. Für Hathor als Herrin von Hu vgl. $L G G$ IV, $103 \mathrm{~b}-104 a$. 
b. Alle drei Ausdrücke dieser Zeile nicht im $L G G$. Der Kopf des Osiris wurde nach vielen Quellen in Abydos bestattet, siehe M. STADLER, „Der Skarabäus als osirianisches Symbol vornehmlich nach spätzeitlichen Quellen“, ZÄS 128 (2001), S. 75-76.

c. Ein Text von fundamentaler Bedeutung ist der Mehithymnus von Edfu (S. Cauville, „L'hymne à Mehyt d'Edfou“, BIFAO 82 [1982], S. 105-109), der die meisten der in Athribis in den Zeilen $x+2-6$ genannten Ausdrücke verzeichnet. Der Nordwind mhyt ist sicherlich eine Anspielung auf die Löwengöttin Mhyt, die Göttin von Mescheich im 8. o.äg. Gau, siehe hierzu den zitierten Mehithymnus (S. 106, Zl. 13-14: is bj hnt mhyt hnt r fnd n sn.s: ,der Ba im Nordwind, der südwärts weht zur Nase ihres Bruders“, vgl. ebenfalls S. 108, Zl. 9-11 sowie den Kommentar von Cauville auf S. 116-118). Cauville weist im Gefolge von Gutbub ebenfalls darauf hin, daß es sich bei der Vereinigung von Schu als Nordwind und Tefnut als Flamme um ein abydenisches Ritual handelt; die einschlägigen Texte wurden von A. Gutbub, Textes fondamentaux de la théologie de Kom Ombo (BdE 47), Le Caire, 1973, S. 446-447 zusammengestellt.

d. Auch diese Zeile sowie alle weiteren dieses Feldes sind dem 8. o.äg. Gau gewidmet. Für Tefnut als Göttin des 8. o.äg. Gaues vgl. S. CAuville, Porte d'Isis, S. 21, 2. In diesem Text findet sich auch ein dem dritten Ausdruck vergleichbare Bezeichnung der Tefnut: mkt su n sut.s: „die den Sohn ihrer Tochter beschützt“; ihre Tochter ist Nut, die Mutter des Osiris. Vgl. auch Edfou VII, 277, 5 irt su $n$ Wsir $m$ T3-wr: „die den Schutz des Osiris im Gau von Abydos bereitet" als Bezeichnung der Mehit, zuvor steht $n b t$ Wbn: „Herrin von Wbn (im 8. o.äg. Gau)“, siehe D. KuRTH, Edfou VII, Wiesbaden, 2004, S. 521, Anm. 4.

e. Für Menet als Bezeichnung der im 8. o.äg. Gau beheimateten Mehit siehe $L G G$ III, 286c 287a. Für das Verhältnis von Menet und Mehit siehe S. CAuville, „L'hymne à Mehyt“, S. 113, Anm. 8.

f. Vgl. hierzu den Namen des Tempels der Mehit pr-Mhyt-wbn: „das Haus der Mehit des Aufgehenden“ (P. Montet, Géographie de l'Égypte ancienne II, Paris, 1961, S. 101) sowie die Bezeichnung der Mehit als nbt Wbn: „Herrin von Wbn (Ortsname im 8. o.äg. Gau)“ in dem verwandten Text Mam. Dendara, 123, 10 (vgl. auch LGG IV, 39b-c). Siehe ebenfalls S. CAuviLLE, „L'hymne à Mehyt", S. 115-116.

g. $L G G \mathrm{~V}, 421 \mathrm{~b}$. Vgl. auch $420 \mathrm{c}$ (hier werden zumindest einige Belege doppeldeutig sein, d.h. sowohl Edfu als auch den Ortsnamen im 8. o.äg. Gau bezeichnen).

h. Vgl. Wb. I, 17, 6 und die folgende Passage aus dem zitierten Mehithymnus (S. CAuville, „L'hymne à Mehyt", S. 108, Zl. 6): ' $n$ Hr tws hmt.t $m$ sht: ,der Räucherarm ist erhoben und deine Majestät ist die Flamme (darin)“.

i. Bislang nicht in der femininen Form belegt, für die Schreibung vgl. LGG III, 211a.

j. Das Verb wbd: „verbrennen“ scheint eine speziell für Mehit charakteristische Vokabel zu sein, vgl. die verschiedenen Ausdrücke in $L G G$ II, 337-339 und den zitierten Mehithymnus.

k. Es ist etwas unsicher, ob dieser Ausdruck noch dem 8. o.äg. Gau zuzuordnen ist (wegen der Göttin Mehit) oder schon dem 9. (wegen des verwandten Textes Mam. Dendara, 123, 12, wo eine mwt Mnw hntyt Hwt-ị h erwähnt wird. Die Erwähnung der mhnyt wrt in Edfou VII, 277, 4 in einem eindeutigen Bezug zum 8. o.äg. Gau (Toponyme Wbn und $T \jmath$-wr) sprechen hingegen stark für die erste Möglichkeit.

1. Für mḥnyt als Bezeichnung der Mehit siehe wieder S. CAuville, „L'hymne à Mehyt“, S. 108, Zl. 1.

m. Mit den İfdwt sind die vier Kobras gemeint, die in der Schlußvignette des pSalt 825 dargestellt sind (Ph. Derchain, Le Papyrus Salt 825 [BM 10051] [MARB 58], Bruxelles, 1965, fig. XIX und S. 188, Anm. 209), die geradezu eine Illustration zu dem Athribistext darstellt. Im einzelnen sind dies noch die Löwin Menet $(x+4)$, die den Nordwind $(m h y t)$ an die Nase des Osiris gibt $(v g l . x+2)$ und Sachmet, die die Flamme gegen dessen Feinde richtet $(v g l . x+4)$. Nicht unwichtig in vorliegenden Zusammenhang ist die Tatsache, daß es im pSalt 825 um eine Beschreibung des Lebenshauses in Abydos geht (VI, 5-6 und der Kommentar von Derchain auf S. 44-46, 48). Für die vier Kobras, die den Abydosfetisch bewachen, siehe den Beitrag von L. Coulon, „Un aspect du culte osirien à Thèbes à l'époque saïte: La chapelle d'Osiris 
Ounefer ,Maître des aliments"“, Égypte. Afrique \& Orient 28 (2003), S. 54-57 (Darstellungen im Hibistempel und auf der Kapelle der Anchnesneferibre in Karnak) mit dem Verweis auf die vier Kobras im oGardiner 363 (R. K. RITNER, „O. GARDINER 363: A Spell against Night Terrors“, JARCE 27 [1990], S. 25-41).

\section{Feld des Hymnus}

Senkrechte Zeile: [YIIII,--WIIIII, ]

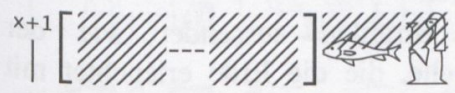

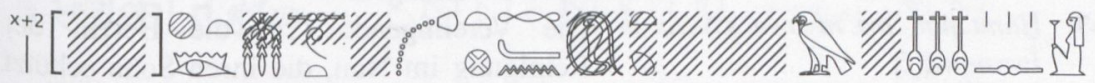

1 [3.

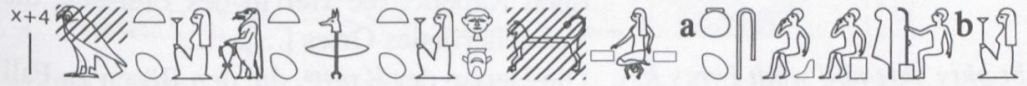

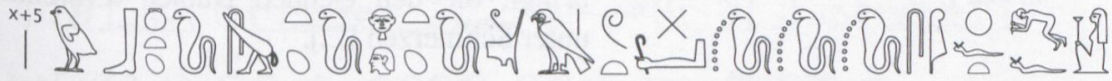

100 [.

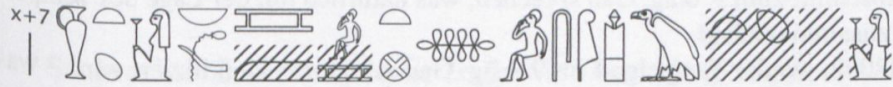

1 [थ

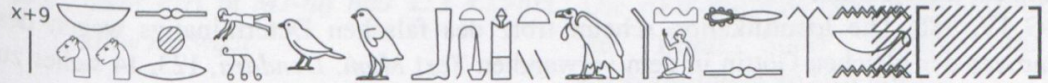

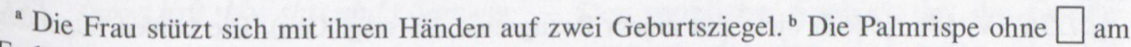
Ende.

Senkrechte Zeile: [...] hr imntt: [...] im Westen.

$\mathrm{x}+1 \quad[\ldots] b s$

$\mathrm{x}+2 \quad[\ldots]$ ht $m s . t i[\mathrm{~m}]$ İpw mhnyt $[\ldots]$ $\operatorname{Hr}[m$ ? $]$ nfrw.s $[\ldots]$.

[...] Sehen ${ }^{\mathrm{a}}$, indem sie geboren wurde in Achmim, die Umringlerschlange, [...] Horus [mit?] ihrer Vollkommenheit. 
$\mathrm{x}+3$ nbty rhyt nbt ťswy ḥnwt $m$ šnw $n$ itn

$\mathrm{x}+4$ bikt špst wsrt hryt-ib nmỉt??

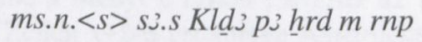

$\mathrm{x}+5$ wbnt sšmwt hryt-tp nt $H r \quad w d t$ nsrwt.s $m$ hfty.f

$\mathrm{x}+6 \quad R^{\complement} t$ wdt mswt mi shty sḥdt ndbw $m$ shty.s

$\mathrm{x}+7$ Hnmt nbt ' $b w \mathrm{w}$ nwn sù su.s $m$ I $w n-m w t[. f]$

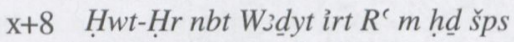
nbt swiš hntyt st [...]

$\mathrm{x}+9$ nbt pḥty shrt nbd wbdt iwnty hsy $m n k n[\ldots]$
Die Herrin der Rechit, die Herrin der beiden Länder, die Gebieterin im Umkreis der Sonnenscheibe ${ }^{b}$.

Das Falkenweibchen, die Prächtige und Mächtige, die inmitten von ...?... ist, nachdem sie ihren Sohn Kolanthes das Kind ${ }^{\mathrm{c}}$ als Verjüngten geboren hat.

Die Aufgehende, die Führende, die Kobra des Horus ${ }^{d}$, die ihre Flammen gegen seinen Feind richtet.

Rat, die Strahlen aussendet wie der Horizontische, die die Erde erleuchtet mit ihren Glanzaugen.

Die Vereinigende (?) ${ }^{\mathrm{e}}$, die Herrin der Reinigung im Nun, die ihren Sohn schützt als Pfeiler [seiner] Mutter.

Hathor, die Herrin von Kom Ischqau (10. o.äg. Gau) $)^{\mathrm{f}}$ das Auge des Re in der prächtigen Kapelle, die Herrin des Preisens, die Vorsteherin des Ortes [...].

Die Herrin der Kraft ${ }^{\mathrm{g}}$, die den Bösen zu Fall bringt, die den elenden Nubier verbrennt unter Schmerzen [...].

a. Vgl. Wb. III, 348, 16-17.

b. $L G G \mathrm{~V}, 208 \mathrm{~b}-c$.

c. Die Erwähnung von Kolanthes zusammen mit der von Achmim in Zeile $x+2$ würde für einen ziemlichen langen Abschnitt zum 9. o.äg. Gau sprechen, was natürlich mit der Lage des Tempels in eben diesem Gau zusammenhängt.

d. Vgl. Edfou IV, 180, 2, wo der Tempelgott im 9. o.äg. Gau mit Horus identifiziert wird - was ein Indiz für die Lokalisierung auch dieser Zeile im 9. o.äg. Gau sein könnte.

e. Die Identifikation der Göttin ist unklar, aber der dritte Ausdruck dieser Zeile in Verbindung mit Edfou I, 307, 13, wo su su.s eine Bezeichnung der Hnmt wrt (LGG VI, 21a-b) ist, spricht immerhin für diese Göttin.

f. $L G G$ IV, 38b. Die Identifikation scheint trotz des falschen Determinativs wegen des Vorkommens der gleichen Göttin in dem verwandten Text Mam. Dendara, 123, 14 sicher zu sein.

g. $L G G$ IV, 58a (jedoch nicht mit einem Bezug zum 10. o.äg. Gau). Die beiden folgenden Ausdrücke sind bislang nicht belegt, allerdings bietet der verwandte Text S. CAuviLle, Porte d'Isis, S. 21, 11 für den 10. o.äg. Gau shrt sbiw $m$ h hst wỉs.f: „,die die Feinde an der Spitze seiner (=Re) Barke zu Fall bringt" doch eine ähnliche Wendung.

\section{Feld des Hymnus}

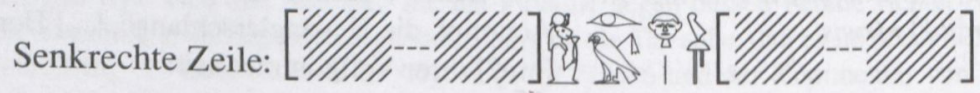




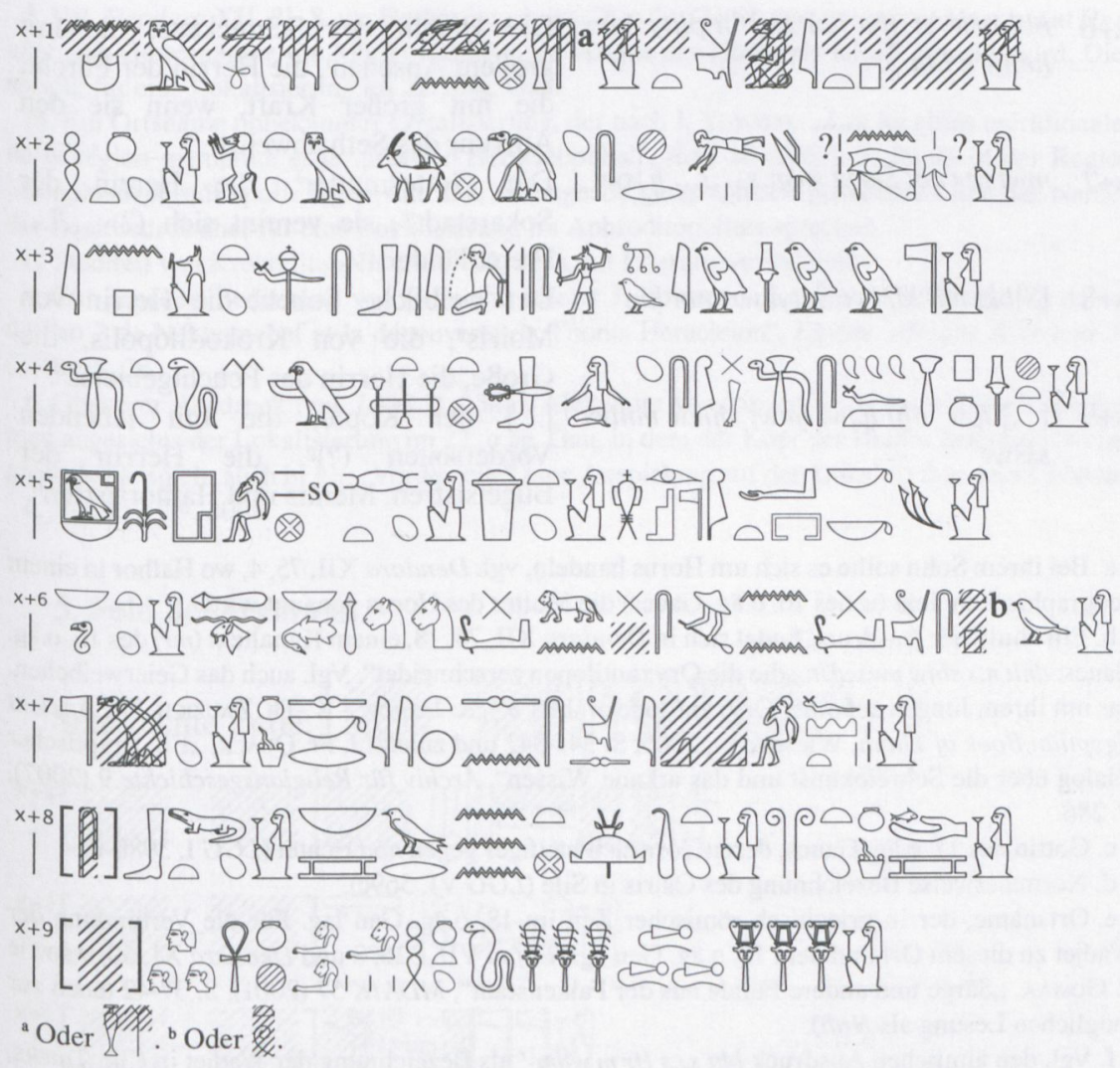

Senkrechte Zeile: [...] Rpwt irt Hr hr imntt: [...] Repit, das Horusauge im Westen.

$\mathrm{x}+1 \quad$ Nhmt- ${ }^{\mathrm{e}}$ wjy $m$ Wnt $[\ldots]$

$\mathrm{x}+2$ hnwt nfrt $m$ Mj-ḥd mkt su.s $<s>h r t$ sbiw $n w$ wdst

$\mathrm{x}+3$ Inpwt hsft sbỉw shrt nbd $\mathrm{r}$ Shmww

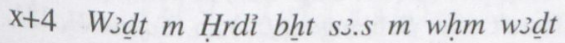
$h^{\prime} w$. f $m$ wid $p n<n>$ Shmt

X+5 Hwt-Hr nbt Hwt-nn-nsw 'dt Bustt su Wsirm list-kskj
Nehemtawai in Hermopolis [...].

Die vollkommene Herrin im Gazellengau, die ihren Sohn ${ }^{a}$ schützt, die die Oryxantilopen zu Fall bringt ${ }^{\mathrm{b}}$.

Der weibliche Anubis ${ }^{c}$, der die Feinde abwehrt, die den, der gegen $S h m w w^{d}$ Böses tut, zurücktreibt.

Wadjet in Hardaie, die ihren Sohn von neuem gebiert $\mathrm{f}^{\mathrm{f}}$, die seine Glieder mit diesem Zepter < der $>$ Sachmet jung hält ${ }^{\mathrm{g}}$. Hathor, die Herrin von Herakleopolis ${ }^{\mathrm{h}}$, das Ichneumonweibchen ${ }^{i}$, Bastet, der Schutz des Osiris auf dem Hügel der $k$ kju-Pflanzen. 
$\mathrm{x}+6 \quad$ nbt nrw '3t šfyt nbt snd 'st phty šn's hnnw $n$ Sth

$\mathrm{x}+7$ mwt ntr nbt Skryt snsn.s ...?... husswt

X+8 [S]bkt nbt $M r-w r \check{S} d t y t w r t ~ n b t ~ h n t$

$\mathrm{x}+9$ [...] tpw 'nht ḥॅwt ḥnwt shmw mniwt $s \check{s ̌} w t$
Die Herrin des Schreckens, die mit großem Ansehen', die Herrin der Furcht, die mit großer Kraft, wenn sie den Aufruhr des Seth abwehrt ${ }^{k}$.

Die Gottesmutter ${ }^{1}$, die Herrin der Sokarstadt $^{\mathrm{m}}$, sie vereint sich (?) ...?... Fremdländer.

Der weibliche Sobek, die Herrin von Moiris $^{\mathrm{n}}$, die von Krokodilopolis, die Große, die Herrin des Feuchtgebietes ${ }^{\circ}$.

[...] der Köpfe, die mit lebenden Vorderseiten (?) $)^{p}$, die Herrin der Bügelsistren, Menits und Hathorsistren ${ }^{q}$.

a. Bei ihrem Sohn sollte es sich um Horus handeln, vgl. Dendara XII, 75, 4, wo Hathor in einem geographischen Text ( $w$ des 16. o.äg. Gaues) die Mutter des Horus genannt wird.

b. Ein ähnlicher Ausdruck findet sich in Dendara XII, 74, 18, einem Kanaltext ( $m r)$ des 16. o.äg. Gaues: dnit n.s sbiw nw wdst: , die die Oryxantilopen zerschneidet“. Vgl. auch das Geierweibchen, das mit ihrem Jungen auf einer Oryxantilope prahlt ( $\left(b^{\prime}\right)$, R. JASNOW - K.-Th. ZAUZICH, The Ancient Egyptian Book of Thoth, Wiesbaden, 2005, S. 341-342 und zuletzt J. Fr. QUACK, „Ein ägyptischer Dialog über die Schreibkunst und das arkane Wissen“, Archiv für Religionsgeschichte 9 (2007), S. 286.

c. Göttin des 17. o.äg. Gaues, deren Zorn sich häufiger gegen $n b d$ richtet (LGG I, 398b-c).

d. Normalerweise Bezeichnung des Osiris in Sile ( $L G G$ VI, 569c).

e. Ortsname, der in griechisch-römischer Zeit im 18. o.äg. Gau lag. Für die Verbindung der Wadjet zu diesem Ort und dem 18. o.äg. Gau vgl. Edfou VII, 326, 8 und Dendara XI, 60, 9 sowie F. GomAÀ, ,,Särge und andere Funde aus der Falkenstadt“, MDAIK 57 (2001), S. 37-42 (auch zur möglichen Lesung als $\mathrm{Hrdi}$ ).

f. Vgl. den ähnlichen Ausdruck bht sủ.s Hr $\mathrm{m}$ whmm-` als Bezeichnung der Wadjet in Chr. THIERS, Tôd II, 244, 5. U. U. wäre die dortige Lücke mit Hilfe des Athribistextes als whm-' $m$ [w’d Shmt zu füllen. Vgl. J. VANDIER, Le Papyrus Jumilhac, Paris, 1961, XIII, 7-10.

g. Vgl. S. Cauville, Porte d'Isis, 32, 1 (swdzt su.s $m$ wud.s.s als Bezeichnung der Wadjet). Vgl. ebenfalls S. SAuneron, Esna III, 291, 22 = S. SAUNERon, Esna V, S. 139: ir w $w d w n w$ Shmt mshnt $m$ 3h-bit: „Die Papyruspflanzen der Sachmet dienen als Geburtsstätte in Chemmis“.

h. Eine der sieben oder zwölf Hathoren ( $L G G$ IV, 102b).

i. Siehe zu dieser Zeile Chr. LeITZ, „Das Ichneumonweibchen von Herakleopolis - eine Manifestation der Bastet“, SAK 38 (2009), S. 161-171.

j. Die Wahl des Substantivs šfyt ist sicherlich geographisch begründet (vgl. den Gott Hry-š.f und $L G G$ VIII, 774a).

k. Eine wichtige Vergleichsstelle ist eine Passage aus des Horusmythos von Edfu (Edfou VI, 121, 1), wo Isis die genannt wird, die den Feind von Naref (im 20. o.äg. Gau) abwehrt (šn't sbỉ r N $z r f)$. In dem gleichen Tableau nimmt Seth die Gestalt einer Schlange ( $h f^{\prime} z$ ) an (Edfou VI, 121, 9 und 10). Dies erinnert einen natürlich an die vorige Zeile $x+5$ mit dem Ichneumonweibchen, das in den zwei geographischen Texten aus Dendara Apophis bekämpft. Es könnte gut möglich sein, daß der Edfutext der dritte Beleg für einen Lokalmythos darstellt, demzufolge Bastet als Ichneumonweibchen Seth/Apophis in Schlangengestalt im 20. o.äg. Gau bekämpft. In dem gleichen Edfutext (Edfou VI, 121, 6-7) wird gesagt, daß dieser Gott (= Horus von Edfu) auf diesem Wasser rudern $(h n)$, kurze Zeit später $(121,9)$ wird das Fest des Ruderns $(h b h n)$ erwähnt. Bei der ägyptischen Vorliebe für Wortspiele könnte die Wahl des Substantivs hnnw in dem Athribistext durch dieses Rudern im 20. o.äg. Gau bedingt sein. 
1. Vgl. Dendara XII, 81, 8, wo Hathor im ph des 22. o.äg. Gaues mwt ntr mwt nt Mnw styt nt Hr m nhn: ,die Gottesmutter, die Mutter des Min, die Amme des Horus als Kind“ genannt wird. Dies spricht für eine Lokalisierung im 22. o.äg. Gau.

m. Ein Ortsname unbekannter Lokalisierung, der nach J. YоYоттE, „Les localités méridionales de la région memphite et le ,pehou d'Héracléopolis'“, RdE 14 (1962), S. 89-93 in der Region Memphis/Aphroditopolis legen soll. Die vorliegende geographisch geordnete Liste der Namen der Repit würde eher für eine Lokalisierung im Aphroditopolites sprechen.

n. Stadtteil von Krokodilopolis ( $L G G$ III, 328a mit Literaturverweisen).

o. Zur genauen Bedeutung von hnt siehe zuletzt J. YoYotTE, „Le second affichage du décret de l'an 2 de Nekhetnebef et la découverte de Thonis Heracleion", Égypte. Afrique \& Orient 24 (2001), S. 28.

p. Obskurer Ausdruck (vgl. LGG II, 154a). Alexandra von Lieven weist mich darauf hin, daß man angesichts der Lokalisierung im 22. o.äg. Gau, in dem der Kopf der Hathor bekanntlich eine große Rolle spielt, auch in hzt: ,Vorderseite“ eine Anspielung auf den Lokalmythos sehen könnte.

q. Vgl. $L G G$ V, $206 a$.

\section{Feld des Hymnus}

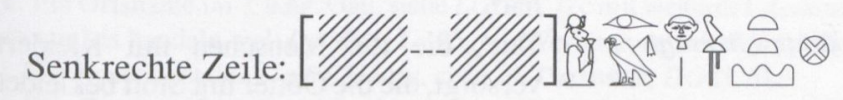

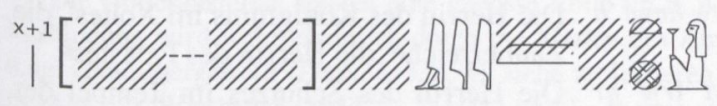

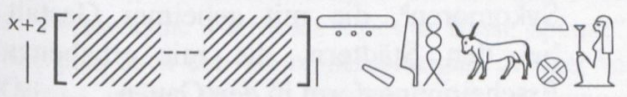
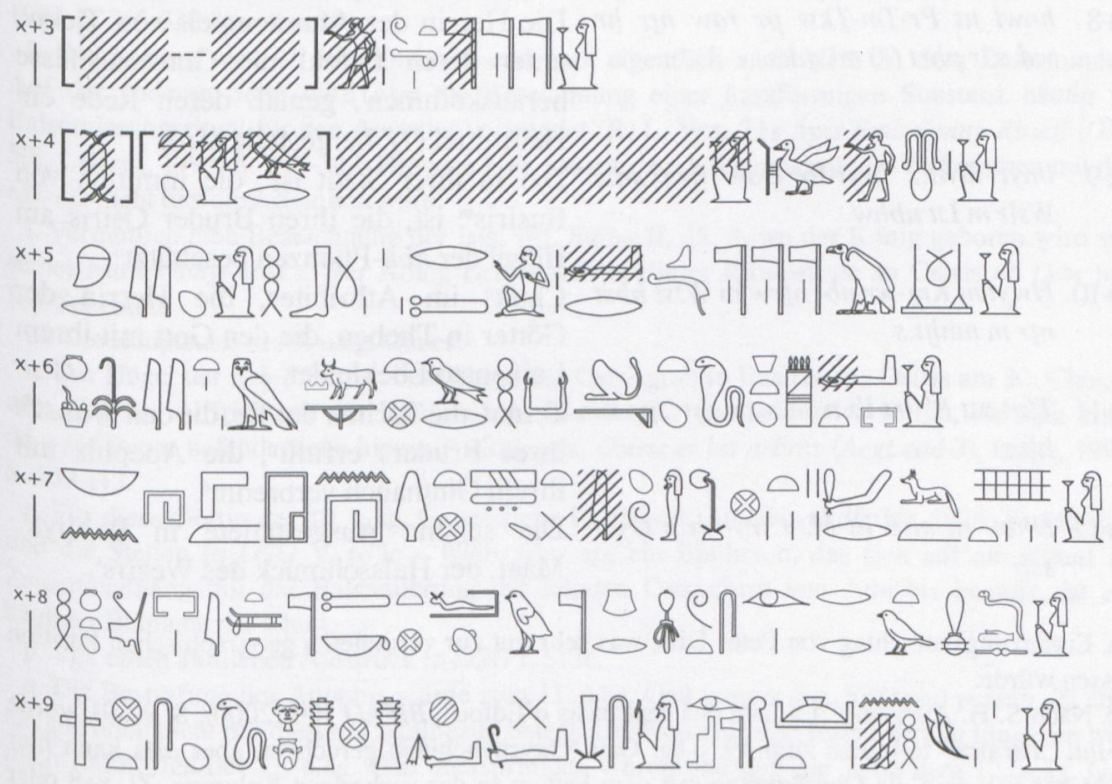


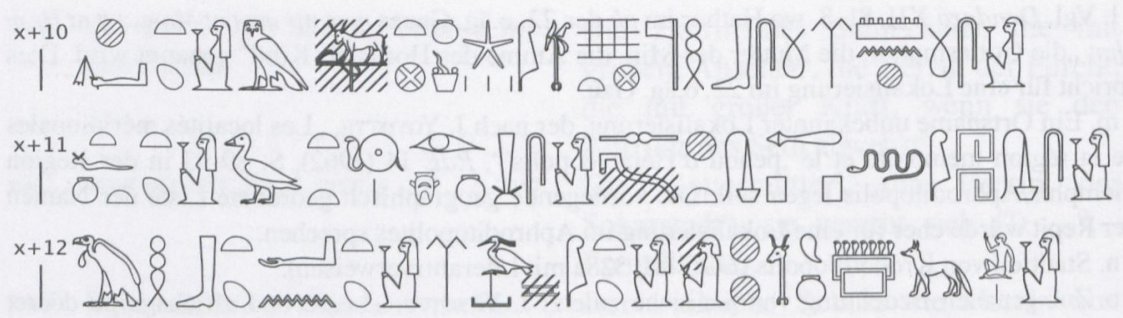

Senkrechte Zeile: [...] Rpwt irt Hr hr imntt: [...] Repit, das Horusauge im Westen.

$$
\begin{array}{ll}
\mathrm{x}+1 & {[\ldots] \text { ilit } m[\text { İit } ?]} \\
\mathrm{x}+2 & {[\ldots] T 3-i h t} \\
\mathrm{x}+3 & {[\ldots] \ldots . . . . \text { wtt } t \text { ntrw }} \\
\mathrm{x}+4 & \text { Nt wrt }[\ldots] \text { kmst Tm }
\end{array}
$$

\section{$\mathrm{x}+5$ Tủit hbst rmt 'rkt nțrw $m$ mnht}

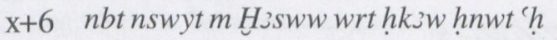

$\mathrm{x}+7$ nbt nht $m$ Hiwt-nhwt št niwtyw dsrt sšt $m$ sp̉̌wt

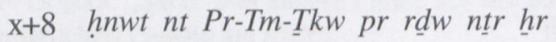
wd.s ir phrt (?) mi $\underline{d} d . s$

$\mathrm{x}+9$ imyt niwt.s ḩryt-ib $\underline{D} d w$ ḩwt sn.s Wsirm ist nbhw

$\mathrm{x}+10$ Hwyt $m$ Km-wr nbt ntrw $m$ W'st ḥbst ntr $m$ mnht.s

$\mathrm{x}+11$ Tfnt sst $R^{\mathrm{C}}$ irt ib n sn.s snwht `spp $m$ hh.s

$\mathrm{x}+12$ Nhbt ' $n t$ m B'ḥt M't iry-ḩh n tzyty $s \jmath b$

\section{[...] die kommt aus [Letopolis] ${ }^{\mathrm{a}}$}

[...] Rinderland ${ }^{\mathrm{b}}$.

[...] ...?..., die die Götter erzeugt hat.

Neith, die Große ${ }^{c}$, [...] die Atum erschaffen hat $^{\mathrm{d}}$.

Taite, die die Menschen mit Kleidern versorgt, die die Götter mit Stoff bekleidet.

Die Herrin des Königtums im Xoites ${ }^{\mathrm{f}}$, die Zauberreiche, die Herrin des Palastes'

Die Herrin des Schutzes im Tempel der Sykomoren $^{\mathrm{h}}$, die mit geheimer Gestalt bei den Städtern, die mit erhabener Erscheinnungsform in den Gauen.

Die Herrin des Atumtempels von Tjeku, unter deren Befehl die Gottesausflüsse herauskommen', gemäß deren Rede ein Heilmittel (?) ${ }^{\mathrm{k}}$ hergestellt wird.

Die in ihrer Stadt ist ${ }^{1}$, die inmitten von Busiris $^{m}$ ist, die ihren Bruder Osiris am Hügel der $n b h$-Pflanzen beschütz $\mathrm{t}^{\mathrm{n}}$.

Chuit $^{\circ}$ im Athribites, die Herrin der Götter in Theben, die den Gott mit ihrem Leinenstoff bekleidet.

Tefnut, die Tochter des Re, die den Wunsch ihres Bruders erfüllt ${ }^{p}$, die Apophis mit ihrem Gluthauch verbrennt ${ }^{\mathrm{q}}$.

Die schöne Ausgestattete in Bakliya ${ }^{r}$, Maat, der Halsschmuck des Wezirss.

a. Ergänzungsvorschlag von Peter Dils, was sehr gut zur vermuteten geographischen Position passen würde.

b. Nach S. H. Aufrère, „La liste des sept oasis d'Edfou“, BIFAO 100 (2000), S. 90-91 wurde T3-iht: „Farafra“ teilweise zum 19. o.äg. Gau (Oxyrhynchites) gerechnet, aber dies kann hier nicht der Fall sein, da Oberägypten mit dem Fayum in der vorherigen Kolumne, Zl. x+8 oder spätestens mit der Zeile danach abgeschlossen ist. Eine Annahme, daß die Oasen zwischen 
Ober- und Unterägypten plaziert wurden, hat keine große Wahrscheinlichkeit. - Sollte jedoch die Ergänzung in Zl. x+1 (Letopolis) richtig sein, so wäre für die Zl. x+2 eine Lokalisierung im 3.

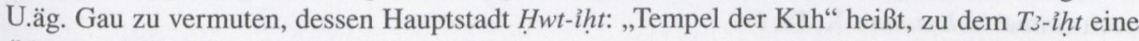
(bislang nicht belegte) Variante sein könnte.

c. Diese Zeile dürfte sich zusammen mit der folgenden auf den 5. u.äg. Gau beziehen (vgl. $L G G$ III, 514a).

d. Ein vor allem in Esna belegtes Epitheton der Neith ( $L G G$ VII, 212b).

e. Für eine ähnliche Schreibung von Tait siehe $L G G$ VII, 359c und H. EL-SAADY, „Reflections on the Goddess Tayet“, JEA 80 (1994), S. 213. Die Lesung der Webergöttin ist sowohl auf Grund der Epitheta wie auch wegen der geographischen Lage (5. u.äg. Gau) sicher.

f. Für das 7000 Jahre währende Königtum des Amun in Xois, das auf einer graphischen Ähnlichkeit zwischen dem Stadtnamen und dem Zahlzeichen für 7000 beruht, siehe S. SAUNERON, Villes et légendes d'Égypte (BdE 90), Le Caire, 1983, S. 171-174 und D. MEEKs, Mythes et légendes du Delta d'après le papyrus Brooklyn 47.218.84 (MIFAO 125), Le Caire, 2006, S. 7 und 183. Im vorliegenden Text wurde das lange Königtum dann auf eine Göttin (Mut?) übertragen ähnlich wie im Thothbuch auf ein Geierweibchen, siehe R. JASNOW - K.-Th. ZAUZICH, Book of Thoth, S. 348-350 und zuletzt J. Fr. QuACK, ,Ein ägyptischer Dialog“, S. 287.

g. Einige Male eine Bezeichnung der Wrt-ḥk $w$ ( $L G G \mathrm{~V}, 171 \mathrm{~b})$.

h. Ein Ortsname im 7. u.äg. Gau, siehe $L G G$ V, $72 \mathrm{c}$ mit weiterer Literatur. Bei der Göttin wird es sich um Isis handeln, vgl. Dendara I, 125, 6 in Verbindung mit Edfou III, 257, 13-18.

i. Die Hauptstadt des 8. u.äg. Gaues, siehe H. GauthiER, DG II, 61.

j. Die Gottesausflüsse spielen eine gewisse Rolle im 8. u.äg. Gau (vgl. $L G G$ VII, 134a). Die Verbindung wird wahrscheinlich durch die Maden des Atum (Dmw Tm) als Gottheiten des 8 . u.äg. Gaues hergestellt (Edfou I, 332, 4), siehe zu letzteren H. BeInLICH, Die „Osirisreliquien“ ( $\ddot{A g}$ Abh 42), Wiesbaden, 1984, S. 251-252 und L. PANTALACCI, „Une conception originale de la survie osirienne d'après les textes de Basse Époque“, GM 52 (1981), S. 61-62. Vgl. zum ganzen Komplex auch den zusammenfassenden Artikel von J. KetTeL, „Canopes, rdw.w d'Osiris et Osiris-Canope“, dans C. Berger u.a. (eds), Hommages à Jean Leclant III (BdE 106), Le Caire, 1993, S. 315-330 über die $r d w$.

k. Die Lesung phrt ist sehr unsicher und paßt eigentlich auch nicht zu dem Determinativ. Auf der anderen Seite wird phrt als Bezeichnung einer harzförmigen Substanz häufig im Balsamierungsritual für den Apisstier verwendet (R. L. Vos, The Apis Embalming Ritual [OLA 50], Leuven, 1993, S. 348-349; Hinweis J. Fr. QuACK), so daß ein paralleles Vorkommen mit den rdw nt tr nicht unwahrscheinlich wäre.

1. Vermutlich eine Bezeichnung der Isis, vgl. Edfou II, 48, 8, wo der König geboren wird von seiner Mutter imyt-niwt.s. Der König richtet sich in dieser Ritualszene an Osiris $n b \underline{D} d w$ und Isis-Schentait.

m. Die Hauptstadt des 9. u.äg. Gaues.

n. Der Hügel der $n b h$-Pflanzen bezeichnet das Osirisgrab in Busiris, wo Osiris am 30. Choiak, dem Tag des Aufrichtens des Djedpfeilers, in einer Krypte beigesetzt wurde, die sich unter dem išd-Baum befand. Siehe hierzu P. Ковмотн, Osiris et les arbres (AegLeod 3), Liège, 1994, S. 109-113.

o. Für diese Göttin des 10. u.äg. Gaues siehe P. Vernus, Athribis (BdE 74), 1978, S. 440-444 und die Stellen in $L G G$ V, 675c - 676b. hbst ntrr, ein Epitheton, das sich auf ein Ritual im Zusammenhang mit der Balsamierung der lokalen Osirisform von Athribis bezieht, ist ein häufiger Beiname der Chuit.

p. Vgl. einen ähnlichen Ausdruck in $L G G$ I, 513c.

q. Die Bestrafung des Apophis würde zum 11. u.äg. Gau (einem sog. Sethgau) passen, ist aber bislang noch nicht mit diesem Lokalbezug belegt. Die Nennung der Tefnut spricht hingegen weit mehr dafür, daß der 11. u.äg. Gau übersprungen wurde und daß die Zeile dem 12. u.äg. Gau (Sebennytes) zuzuordnen ist (vgl. Dendara XII, 194, 9). 
r. Nhbt-' $n t$ ist in den wenigen Belegen stets ein Beiname der Nhmt- ${ }^{-} w 3 y$ (vgl. $L G G$ IV, 276c 277a), was zu $B$ cht , der Hauptstadt des 15. u.äg. Gaues paßt. Die Verbindung mit Maat erscheint noch einmal in Dendara X, 58, 8-9.

s. Bislang 9 Belege in $L G G \mathrm{I}, 411 \mathrm{a}$, die zumeist in Verbindung mit Maat stehen, siehe hierzu mit einigen Abbildungen und zahlreichen Stellen B. GRDSELOFF, „L'insigne du grand juge égyptien“, ASAE 40 (1940), S. 185-207.

\section{Feld des Hymnus}

Senkrechte Zeile: [
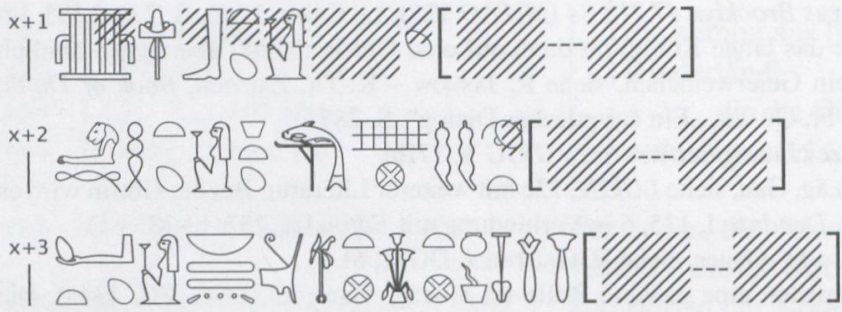

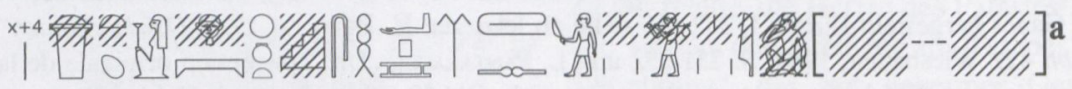

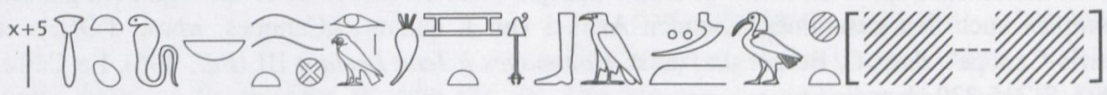

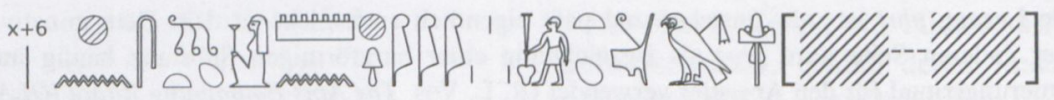

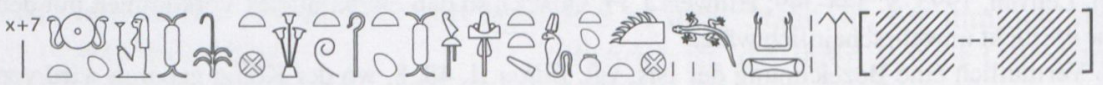

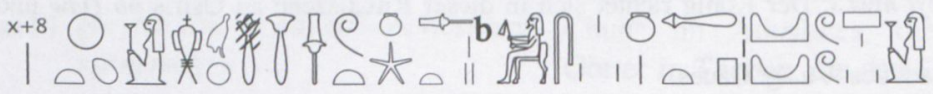

1 -

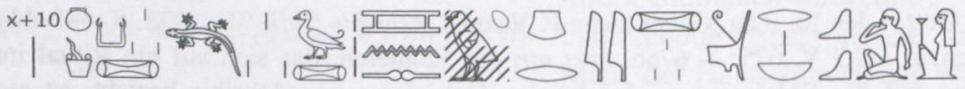

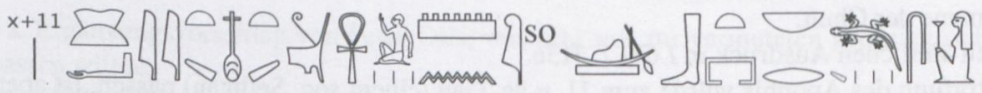

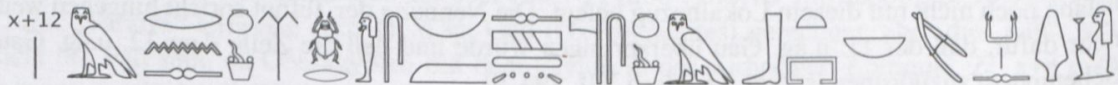

a Der Vorschlag von Joachim Quack (hзtyw šmsyw r-ht.s) scheint sich zu bestätigen. 
${ }^{\mathrm{b}}$ Das Zepter steht für

Senkrechte Zeile: [...] Rpwt irt Hr hr imntt: [...] Repit, das Horusauge im Westen.

$\mathrm{x}+1$ hntyt $[\ldots]$

$\mathrm{x}+2$ Hst-mhyt hinwt Hst-mhyt $n d m t$ sty $[\ldots]$

$\mathrm{x}+3$ Mwt nbt tzwy $m$ Wist-Mhw nbt smj-tวwy [...]

$\mathrm{x}+4$ Bustt hryt hndw.s h'py $m$ phr.s ḩstyw šmjyw r[ht.s ...]

$\mathrm{x}+5$ W3dt nbt Imt îrt Hr bnrt mrwt wb3t mis $m$ sht [...]

X+6 Hnst mnht shrw smswt nt Hr isbtt $[\ldots]$

X+7 nswt-bityt nt Šm $m^{e} w \quad M h w^{\mathrm{i}}$ hkst nt imntt ỉbtt Rnn-wtt nt $K m t$ šst ksw m [...]

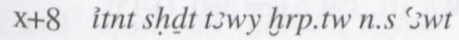

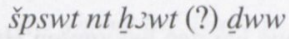

$\mathrm{x}+9$ nbt nsrt 'st phty sft.tw n.s ihhw spdw wsh h้swwt.s m mnt $n t r^{\prime} n b$

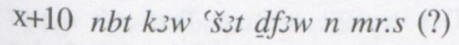
irt hrt n rз nb wnm

$\mathrm{x}+11$ h'yt nfrt nt 'nhw mni bw nb $r$ dmi.s

$\mathrm{x}+12 m$ rn.s $n b \quad m$ hprw.s $n b m$ sšt ’.s nb $m$ bw nb mr kj.s im
Die Vorsteherin von [...].

Hatmehit $^{\mathrm{a}}$, die Herrin des 16. u.äg. Gaues, die mit angenehmem Duft ${ }^{\mathrm{b}}$ [...].

Mut, die Herrin der beiden Länder im Theben Unterägyptens $^{c}$, die Herrin der Vereinigung der beiden Länder, [...].

Bastet, die auf ihrem Thron ist ${ }^{\mathrm{d}}$, in deren Umkreis der Nil ist ${ }^{\mathrm{e}}$, in deren Gefolge die Messer- und Wanderdämonen sind ${ }^{\mathrm{f}}[\ldots]$.

Wadjet, die Herrin von Buto ${ }^{\mathrm{g}}$, das Horusauge mit großer Beliebtheit, die den Blick öffnet mit dem Glanzauge [...].

Chensit ${ }^{\mathrm{h}}$, die mit trefflichen Plänen, Die Älteste des Horus des Ostens [...].

Die Königin von Ober- und Unterägypten, die Herrscherin des Westens und des Ostens, die Renenutet Ägyptens, die mit zahlreichen Speisen in $[. .$.$] .$

Die Sonnenscheibe, die die beiden Länder erhellt, der man die prächtigen Edelsteine der Steinbrüche (?) und Berge darbringt'.

Die Herrin der Flamme mit großer Kraft, der man Rinder und Vögel schlachtet, deren Opferaltäre täglich geschmückt werden.

Die Herrin der Speisen mit vielen Nahrungsmitteln für den, den sie liebt, die den Bedarf für jeden essenden Mund erschafft.

Die schöne Wohnstätte der Lebenden, an deren Häfen jedermann anlandet ${ }^{k}$

in jedem ihrem Namen, in jeder ihrer Erscheinungsform, in jeder ihrer Gestalt, an jedem Ort, an dem ihr Ka zu sein wünscht'.

a. In Dendara I, 127, 9 ebenfalls die Bezeichnung der Tempelgöttin im 16. u.äg. Gau. Siehe zum letzten Stand der Diskussion um die Identifizierung des heiligen Tieres der Hatmehit (Delphin oder Schilbe) J. Yoyotte, in P. Vernus - J. YoyotTe, Bestiaire des pharaons, Paris, 2005, S. 240-242 und 757, der sich (bezeichnenderweise unter dem Stichwort ,Dauphin') auf Grund von Funden mit Gräten der Schilbe in Mendes für diesen Fisch ausspricht (siehe hierzu A. D. DE RoDRIGo, in: Bulletin de liaison du Groupe internationale d'étude de la céramique égyptienne 21 [2000], S. 7-12, die weitere Untersuchungen ankündigt). Der letzte Beitrag stammt von Chr. ZIVIE-Coche, „Hatmehyt, le tilapia, le lotus et le Ba de Mendès“, in W. Claes (Hgg), Elkab and Beyond. Studies in Honour of Luc Limme (OLA 191), Leuven, 2009, S. 545-557, die vorschlägt, daß es sich bei dem 
Tier des Gauzeichens ursprünglich um einen Delphin gehandelt haben könne (von ihr selbst als zweifelhaft erachtet), der später uminterpretiert wurde in eine Tilapia.

b. Dies ist kein seltener Ausdruck ( $L G G$ IV, 602b), aber der Lokalbezug zum 16. u.äg. Gau war bislang unbekannt.

c. Tell el Balamun, die Hauptstadt des 17. u.äg. Gaues. In Dendara I, 127, 12 wird ebenfalls Mut im 17. u.äg. Gau genannt. Zahlreiche weitere Stellen sind erhältlich in dem Kapitel über $S m \jmath-B h d t$ bei I. Guermeur, Les cultes d'Amon hors de Thèbes (BEHE SR 123), Turnhout, 2005, S. 202-245.

d. $L G G \mathrm{~V}, 440 \mathrm{~b}$. Mit diesen beiden Informationen bezieht sich die Zeile klar auf den 18. u.äg. Gau.

e. Dies ist die sechste Stelle, die auf die besondere Lage des von zwei Kanälen umschlossenen Heiligtums der Bastet anspielt (Herodot II, 138), siehe hierzu die Diskussion bei D. KuRTH, Edfou VII, Wiesbaden, 2004, S. 495, Anm. 10 mit der älteren Literatur (am wichtigsten S. SAUNERON, „Villes et légendes. VI. À propos du „toponyme“ Achérou (Ǐšrw)“, BIFAO 62 [1964], S. 50-57, der auch schon zwei der drei Edfustellen zitiert). Siehe auch den Kommentar von A. B. LLoyD, Herodotus Book II, Commentary 99-182 (EPRO 43), vol. 3, Leiden, 1988, S. $94-95$ (zu den im 19. Jhd. noch sichtbaren archäologischen Spuren der beiden Kanäle). Eine weitere wichtige Stelle (mit einer Teilparallele in Dendara XII, 199, 4) ist die Erwähnung im Brooklyner Deltapapyrus (IX, 3-5), siehe D. MeEks, Mythes et légendes, S. 20 und S. 240-241.

f. Spontaner Vorschlag von Joachim Quack auf der 3. Ptolemäischen Sommerschule am 13. 8. 09. g. Für Wadjet in Verbindung mit dem 19. u.äg. Gau siehe z.B. LGG II, 271 b.

h. Siehe zu dieser Göttin des 20. u.äg. Gau die in $L G G$ V, 761b-c genannten Stellen und Literatur. i. Trotz des nachfolgenden imntt i $3 b t t$ die wahrscheinlichere Lesung (statt rsy mhty), vgl. insbesondere Dendara II, 68, 6 (nswt nt $\breve{S}^{\prime}{ }^{\prime} w$ bityt $n t$ Mḥw hkst $m$ imntt l̉btt). Für weitere Stellen siehe $L G G \mathrm{~V}, 539 \mathrm{a}$.

j. Für einen ähnlichen Ausdruck vgl. $L G G$ V, 948b-c (Edfou II, 116, 11), dessen zweifelnde Lesung durch den Athribis-Text eine gewisse Bestätigung erfährt. Die obige Übersetzung geht ferner von einem Fehler von ` für $h$ aus (vgl. für letzteres $W b$. III, 359, 6 und 360, 11-15).

k. Hinweis J. Fr. Quack.

1. Es handelt sich hierbei um nahezu die gleiche Schlußformel wie bei den bekannten Hymnen aus Esna, siehe S. SAUNERON, L'écriture figurative dans les textes d'Esna (Esna VIII), Le Caire, 1982, S. 12 (vgl. auch J. Fr. QUACK, „Ein neuer funerärer Text der Spätzeit (pHohenzollernSigmaringen II)“, ZÄS 127 [2000], S. 78, Kol. 1, 5). Im Unterschied zu Esna sind hier jedoch die Namen der Gottheit nicht kryptographisch geschrieben; zudem findet sich keine vergleichbare geographische Reihung bei den Litaneien aus Esna. Der einzige weitere Text mit dieser Schlußformel stammt ebenfalls aus Athribis; auf der genau gegenüberliegenden Seite (L 3) finden sich die Namen der Bastet.

\section{Feld des Hymnus}

\section{Senkrechte Zeilen:}
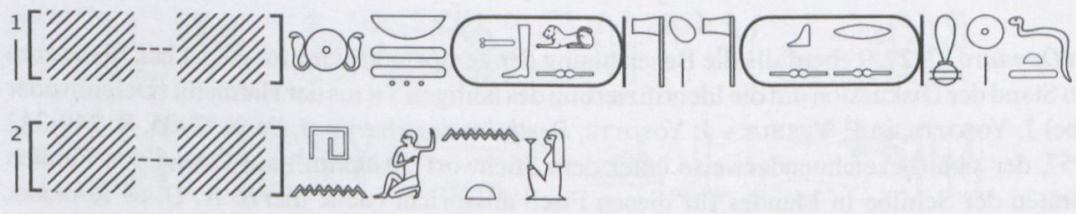

$\stackrel{x+1}{\text { | }}$ völlig zerstört $]$ 


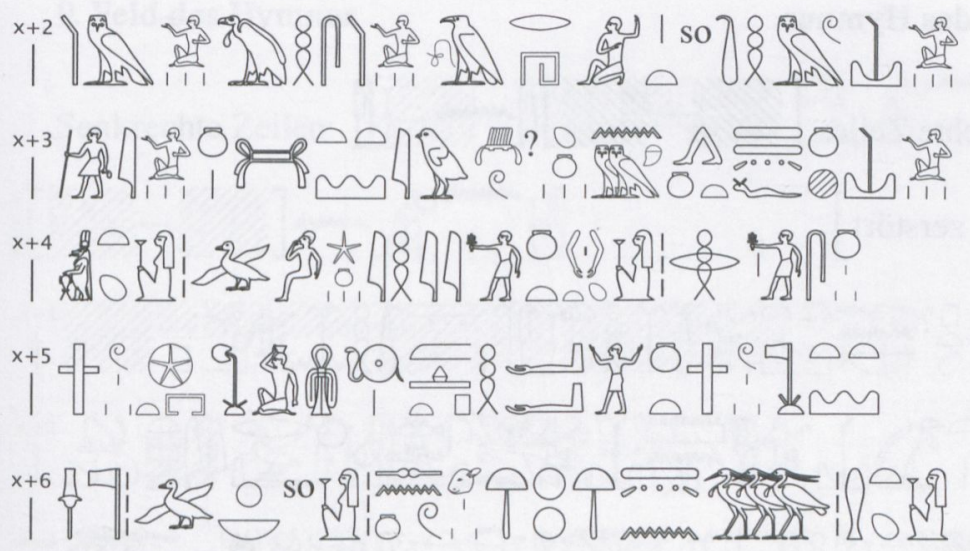

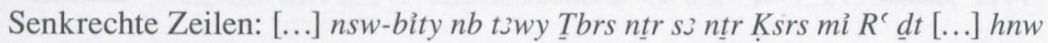
$n . t:$, ,[...] der König von Ober- und Unterägypten Tiberius, der Gott, Sohn eines Gottes Kaisaros mit Re ewiglich, [...] Jubel für dich“".

$\mathrm{X}+1 \quad[\ldots]$

$\mathrm{x}+2$ `mw Nhsyw wrh n.t Tmhw

$\mathrm{x}+3$ srw nw Stt $r$ jw.sn nmt n.t und alle Fürsten von Asien ${ }^{\mathrm{a}}$. Es kommen zu dir die t3wy Fnhw

$\mathrm{x}+4$ špswt hִn $\underline{h}$ rdw.sn $i h y(w)$ nw shnwt hn' ihyw.sn

$\mathrm{x}+5$ imyw dwst hft $R^{\mathrm{e}} m$ htp $h^{\mathrm{e}}$ n.t imyw hrt-ntr

$\mathrm{x}+6$ shmw hn' pswtyw sn hptyt' $n$ bjw hmt.t

\section{[...]}

die Asiaten und die Nubier. Es tanzen für dich die Libyer beiden Länder Phönikiens.

Die Monatsgöttinnen zusammen mit ihren Kindern, die Musikanten der Begleiterinnen zusammen mit

Die in der Unterwelt sind, befinden sich vor Re beim Untergang ${ }^{\mathrm{c}}$. Es jubeln zu dir die, die in der Nekropole sind,

die göttlichen Mächte und die Urzeitlichen. Die Welt (d.h. ihre Bewohner) verehren die Macht deiner Majestät. den Musikanten ${ }^{\mathrm{b}}$.

a. Die tanzenden Fremdvölker sind auch in einem bekannten Hymnus aus Medamud für $R^{\prime} t$-t wwy als Hathor belegt, der anläßlich der Feierlichkeiten bei der Rückkehr der fernen Göttin rezitiert wurde; siehe die letzte Bearbeitung von J. C. DARNELL, „Hathor returns to Medamud“, SAK 22 (1995), S. 64-80.

b. Gemeint sind die thoerisgestaltigen Monatsgöttinnen, siehe hierzu D. Mendel, Die Monatsgöttinnen in Tempeln und im privaten Kult (RitesEg XI), Turnhout, 2005. Der Name shnt war bislang nur im Singular auf der Thoerisstatue Kairo CG 39147 bekannt (D. Mendel, Die Monatsgöttinnen, S. 31). Nach den Ergebnissen von Mendel handelt es sich um vier verschiedene Sets von Gottheiten (S. 77-92): ein Monatsgott, eine Monatsgöttin, ein Kindgott und eine Schutzgöttin. Das in dieser Zeile belegte System in Athribis differiert davon ein wenig. Die špswt sind sicher die Monatsgöttinnen, die shnwt die Schutzgöttinnen. Die hrdww werden die Kindgötter sein, aber neu sind die zweimal genannten ỉhyw.

c. Siehe $L G G$ I, $286 \mathrm{c}$ mit zahlreichen ähnlichen Stellen. 


\section{Feld des Hymnus}

Senkrechte Zeile: [WIIIII,--1IIIIII, $]$ W $\stackrel{x+1}{\mid}[$ völlig zerstört $]$
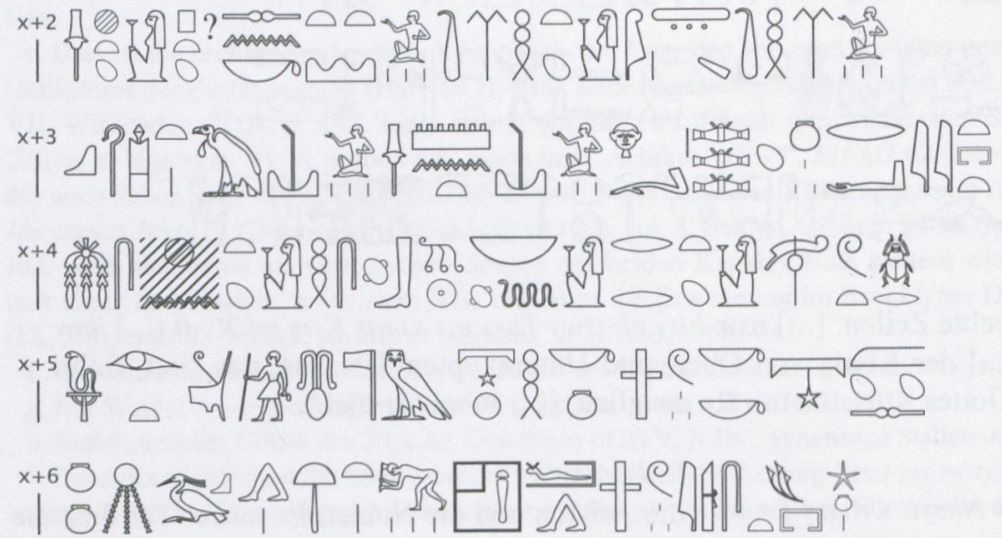

Senkrechte Zeile: [...] i n.t. ,„[...] für dich“.

$\mathrm{x}+1 \quad[\ldots]$

$\mathrm{x}+2$ Shmt hnw Sttyw Tmht $m$ T3-Tmhw

$\mathrm{x}+3$ hkıt liwntyw hrp Mntyw hr rmn g’wt.sn $r$ 'syt.t.

X+4 Mshnt hsbt 'ḥ'wnbt šsy rrt wdt hpr

x+5 ỉ bt nt Tm $m$ grḥ ḥkn imyw-kkw m muj.s

$\mathrm{x}+6$ wbnt 'k šwwt htpt pr imyw-is.sn
[...].

Sachmet, die Herrin der Asiaten (?) ${ }^{\mathrm{a}}$, die Libyerin im Land der Libyer ${ }^{\mathrm{b}}$.

Die Herrscherin der Bogenvölker ${ }^{\mathrm{c}}$, die die Asiaten leitet, indem sie ihre Abgaben zu deinem Heiligtum tragen.

Die Geburtsgöttin, die die Lebenszeit berechnet, die Herrin des Schicksals und der Erziehung, die das befiehlt, was geschieht ${ }^{d}$.

Das linke Auge des Atum in der Nachte: Die in der Finsternis jubeln bei seinem Anblick.

Die leuchtet und die Schatten treten ein, die untergeht und die in ihren Gräbern kommen heraus ${ }^{\mathrm{f}}$.

a. Es ist unklar, ob hier die Sttyw: „Asiaten“ oder die Styw: „Nubier“ gemeint sind.

b. Vgl. $L G G$ V, 215b für Sachmet als Herrin der Libyer.

c. Vgl. $L G G$ V, 65b Hwt-liwntyw: ,die die Bogenvölker schlägt“ als Bezeichnung der Sachmet.

d. Für einen sehr ähnlichen Text vgl. Dendara II, 149, 3-4. Der letzte Ausdruck ist ebenfalls typisch für Mshnt, vgl. $L G G$ II, 641c.

e. Eine Bezeichnung der Wadjet ( $L G G$ I, 104a). Gemeint ist letztendlich der Mond.

f. Lesungsvorschlag von Philippe Collombert und Laurent Coulon auf der 3. Ptolemäischen Sommerschule. 


\section{Feld des Hymnus}

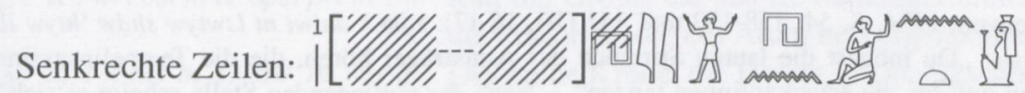
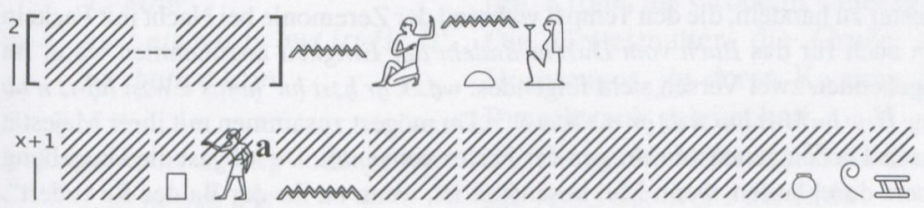

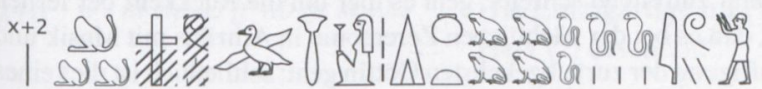

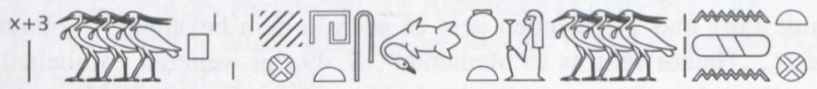

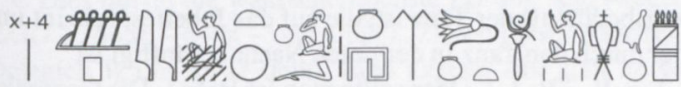
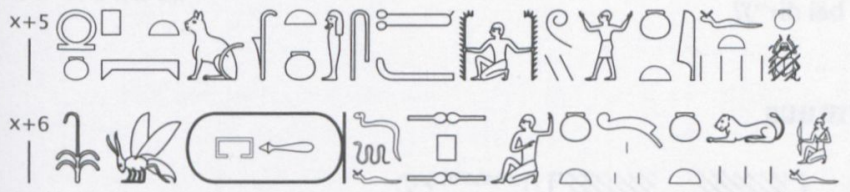

"Von der Figur sind nur die Beine erhalten.

Senkrechte Zeilen: [...] hy hnw n.t [...] hnw n.t: ,„[..] Jauchzen und Jubel für dich. [...] Jubel für dich".

$\mathrm{x}+1 \quad[\ldots]$

$\mathrm{x}+2$ ntrw imyw pswt di n.t Hmnyw ỉsw

$\mathrm{x}+3$ bjw $P<m>$ hy snd n.t bjw Nhn

$\mathrm{x}+4$ šspw dhn nhm n.t ¿̉wtyw shd

$\mathrm{x}+5$ šnw n pt mi kd.s thh n.t ifdwt nt tว

x+6 nsw-bity $\operatorname{Pr}$ - 3 ds.f sps n.t ns (?) n.t imyw-ru $m \breve{s}^{e} \cdot f$
[...]

Götter, die in der Urzeit sind ${ }^{\mathrm{a}}$. Die Achtheit gibt dir Lobpreis.

Die Bas von Buto jubeln (?) $)^{\mathrm{b}}$ und die Bas von Hierakonpolis haben Respekt vor dir.

Der Chor der Taktangeber': es jubeln dir die Amtsträger zu, die den Tempel erleuchten ${ }^{\mathrm{d}}$. Der Umkreis des ganzen Himmels: es jubeln für dich die vier Seiten der Erde.

Der König von Ober- und Unterägypten, der Pharao selbst tanzt für dich. Die Generäle seines Heeres kommen (?) $)^{\mathrm{e}} \mathrm{zu}$ dir.

a. Vgl. $L G G$ IV, 461b (Ntrrw-imyw-pswt.sn). Die Schreibung für pswt ist ungewöhnlich; die für die nachfolgende Achtheit wohl singulär, auch wenn die Lesung völlig sicher erscheint.

b. Bei einer Lesung von hy snd stünden die bjw $P$ isoliert, aus diesem Grund wurde die Präposition ergänzt. Das $s$ ist der Anlaut von snd, vgl. ähnliche Schreibungen in P. WILson, $A$ Ptolemaic Lexikon (OLA 78), Leuven, 1997, S. 878-879. 
c. Wb. IV, $537,12$.

d. Eine sehr ähnliche Stelle findet sich bei Fr. R. HeRBIN, Le livre de parcourir l'éternité (OLA 58), Leuven, 1994, S. 54, 158-159 und 441 (III, 16-17): sdm.k dniwt nt ljwtyw shdw Chtyw ibjw $n$ liwntyw: „Du mögest die lauten Stimmen der Amtsträger hören, die die Tempelinsassinnen erhellen und für die Musikantinnen tanzen“. - Nach der vorliegenden Stelle scheint es sich bei den $i$ w wyw um Priester zu handeln, die den Tempel während der Zeremonie bei Nacht mit Fackeln erhellen, was dann auch für das Buch vom Durchwandeln der Ewigkeit anzunehmen wäre. Im

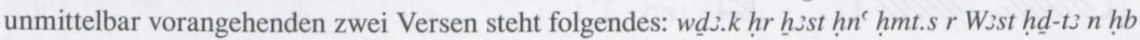

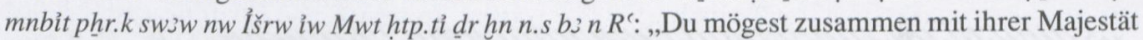
durch die Wüste nach Theben gehen am Morgen des Festes von mnbit. Du mögest die Umgebung des Ischerugewässers durchlaufen, wenn Mut besänftigt ist, wenn zu ihr der Ba des Re rudert“. Wie Herbin in seinem Kommentar zutreffend schreibt, geht es hier um die Rückkehr der fernen Göttin und man darf vermuten, daß es bei der nächtlichen Zeremonie in Athribis mit Musik und Fackeln ebenfalls um die Besänftigung der zurückgekehrten Göttin geht; schließlich ist dies eines der Hauptthemen des Repittempels (vgl. Athribis II, C3, 98-99; C5, 40-42). Um die nächtliche Besänftigung der Hathor mit Tanz und Trunkenheit geht es auch in dem bekannten Hymnus aus Medamud (J. C. DARnELl, „Hathor returns to Medamud“, S. 49 mit weiteren Parallelen):

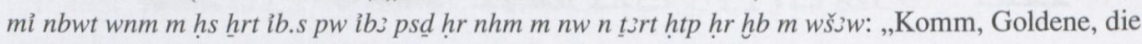
sich vom Lobpreis ernährt, deren Herzensbedürfnis der Tanz ist, die auf das Jubelfest scheint im Augenblick des Fackelanzündens (?), der durch den Tanz in der tiefen Nacht besänftigt ist“"

e. Die Lesung ist fraglich. Für $n s$ vgl. Wb. II, 321, 1-2. Oder sollte man ' $r$ k lesen (,Die Generäle seines Heeres schwören bei dir")?

\section{Feld des Hymnus}
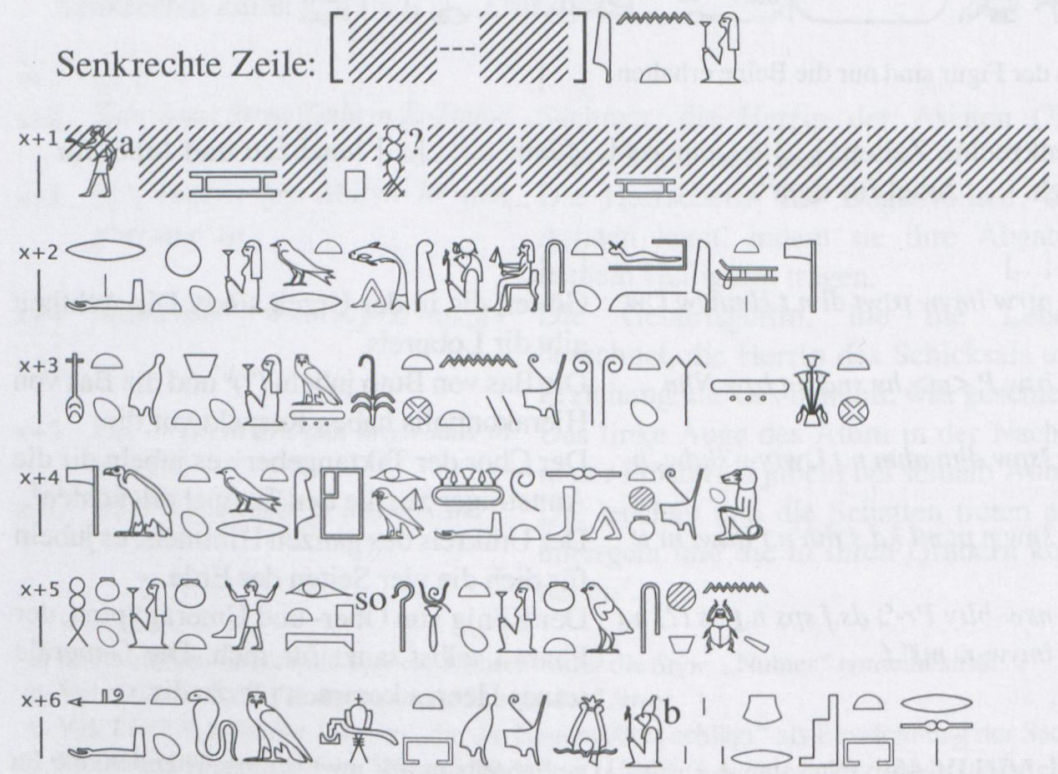

${ }^{\mathrm{a}}$ Von der Figur sind nur Beine erhalten. ${ }^{\mathrm{b}}$ Der Gott hält statt des Zepters eine Geißel in der Hand.

Senkrechte Zeile: [...]i n.t: , ,[...] für dich“. 
$\mathrm{x}+1 \quad[\ldots]$

$[\ldots]$.

$\mathrm{x}+2 R^{\prime} t$ wrt bst $m R^{\prime}$ špst prt $m$ Tm Rat, die Große, die aus Re herausgekommen ist, die Prächtige, die aus Atum hervorgekommen ist.

$\mathrm{x}+3 \quad$ nfrt hnnt $m$ T3-šm $n t$ wrt $m$ Die weiße Krone, die Herrin in Oberägypten, die $T 3-M h w$ rote Krone, die Große in Unterägypten.

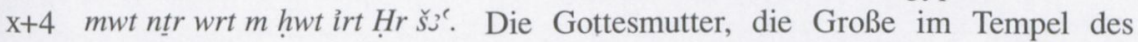
tw sbt th $n$ kj.s Horusauges, zu deren Ka man am Anfang den Rauschtrank geleitet hat ${ }^{\mathrm{a}}$.

X+5 hnwt nt $k j\{i \mathfrak{i}\}<n>p t h k j t$ Die Herrin der Höhe des Himmels, die Herrscherin iswt nt wshnt des Amts der Weite der Erde

$\mathrm{x}+6 \quad w^{\prime} t m$ stp-s3 $h^{\prime} n b \underline{h} r$ st-r3.s $\quad$ Die Einzigartige im Palast, unter deren Befehl der Herr (?) erscheint ${ }^{c}$.

a. Zur Lesung des Pavians vgl. die Ausdrücke in $L G G$ VII, $15 \mathrm{c}$.

b. ir vor $p t$ dürfte falsch sein; ob $i s w t$ nach $h k$ j richtig ist fraglich (vgl. Dendara X, 216, 13-14), aber möglich ( $L G G \mathrm{~V}, 538 \mathrm{a})$.

c. Dies dürfte die wahrscheinlichste Lösung sein (wegen der Häufigkeit des Ausdrucks - vgl. $L G G \mathrm{~V}, 645 \mathrm{c}-646 \mathrm{a}$ - und Opet I, 21, wo dieser Ausdruck kurz hinter dem bislang nur einmal belegten $W^{\prime} t m$ stp-sj steht). Eine Lesung $n s w$ statt $n b$ wäre jedoch nicht ausgeschlossen (vgl. $L G G \mathrm{~V}, 647 \mathrm{a}-\mathrm{b})$. 